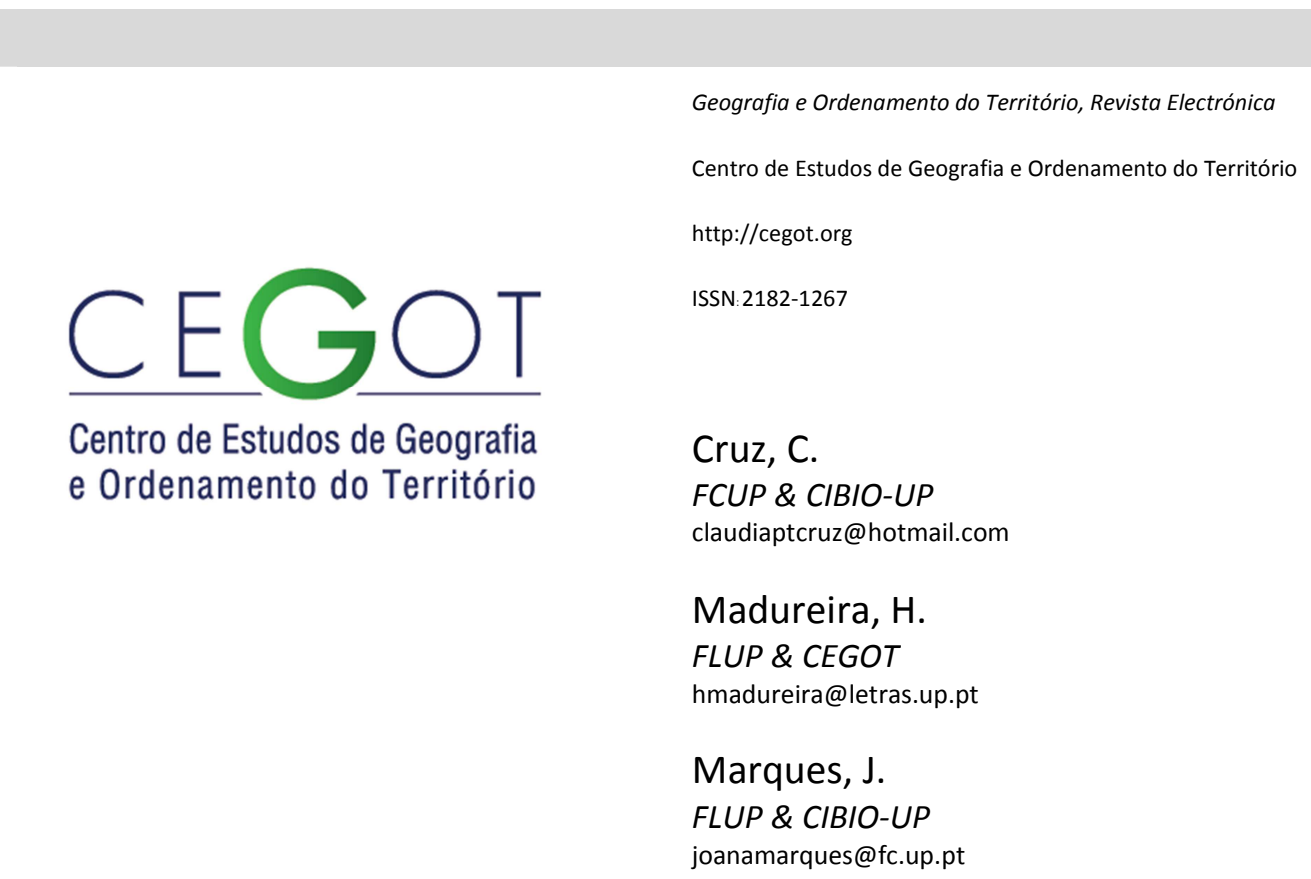

\title{
Análise espacial e estudo da fragmentação da Paisagem da Aboboreira
}

Referência: Cruz, C.; Madureira, H.; Marques, J. (2013). Análise espacial e estudo da fragmentação da Paisagem da Aboboreira. Revista de Geografia e Ordenamento do Território (GOT), n. 4 (dezembro). Centro de Estudos de Geografia e Ordenamento do Território, p. 57-82

\section{Resumo}

$\mathrm{Na}$ atualidade os processos de fragmentação representam uma das maiores preocupações no âmbito da conservação da biodiversidade, já que a heterogeneidade dos fragmentos afeta a dinâmica e o estabelecimento de espécies vegetais e animais. Neste trabalho caracterizamos a evolução e transformação da paisagem da Aboboreira (NO Portugal) através da análise da evolução da ocupação do solo nos últimos 20 anos. Os resultados mostram uma diminuição de manchas agrícolas, com consequente substituição por manchas florestais e urbanas, sendo a expansão urbana mais pronunciada nas áreas de vale. No entanto, o resultado mais relevante aponta para uma fragmentação geral da paisagem com consequente perda de habitats naturais.

Palavras-Chave: Aboboreira, Estatística Kappa, Métricas da Paisagem, Fragmentação, Análise espacial. 


\begin{abstract}
At the present time landscape fragmentation is a major concern for biodiversity conservation, since habitat heterogeneity strongly affect population dynamics and species establishment. In this work we studied the Aboboreira landscape transformation through the analysis of the land cover changes in the last 20 years. Results show that there has been a marked reduction of agricultural land, resulting in an increase of forest and urban landscapes; the urban expansion is particularly evident in valley areas. The most relevant result shows a general fragmentation in Aboboreira region with consequent natural habitat loss.
\end{abstract}

Keywords: Aboboreira, Kappa statistics, Landscape metrics, Fragmentation, Spatial analysis.

\title{
1. Introdução
}

O objetivo deste trabalho é caracterizar a evolução e transformação da paisagem da Aboboreira através da análise da evolução da ocupação do solo nos últimos 20 anos. Pretende-se, designadamente, analisar os padrões espaciais dessa evolução, compreender em que sentido se deram as modificações de ocupação do solo e, sobretudo, determinar se ocorreu fragmentação.

\subsection{Fragmentação}

Em 1980, a The World Conservation Union identificou a fragmentação como uma das principais causas da diminuição da diversidade biológica, e atualmente vários estudos continuam a provar essa realidade (Antwi, Krawczynski, \& Wiegleb, 2008; Olff \& Ritchie, 2002).

A fragmentação de habitats é um processo comum relacionado com a alteração da paisagem e afeta tanto a sua estrutura como função (Botequilha Leitão \& Ahern, 2002), logo, o termo fragmentação é utilizado para descrever um estado ou um processo de alteração de habitats. Enquanto o estado se refere à separação de habitats inicialmente ligados, o processo resulta duma alteração do desenho ou da disposição espacial dos habitats ao longo do tempo, e compreende três componentes espaciais: a) redução no tamanho dos habitats, b) aumento do número de manchas e c) aumento do seu isolamento e perda de conectividade (Hunter, 1996). 
A perda de habitat é comum a todos os processos de fragmentação desse habitat. A divisão de uma área, além de reduzir a sua dimensão, altera a forma natural e o número de manchas dos habitats. Estes processos repercutem-se no aumento da razão entre o perímetro e a área, o que leva a uma maior proporção de habitat situado perto da orla que se torna, por conseguinte, mais vulnerável a perturbações (Hunter, 1996). O isolamento resulta do aumento da distância entre fragmentos.

Diversos autores têm-se dedicado a estudar os efeitos da fragmentação nos processos ecológicos (Fahrig, 2003; Johansson, 2008; Wiens, 1976). As principais consequências identificadas passam pela perda de espécies e por alterações na estrutura das comunidades. A perda de espécies está fortemente relacionada com a redução do tamanho das manchas dos habitats (Belinchón, 2007). A correlação entre o número de espécies e o tamanho das manchas dos habitats assenta em três princípios: a) manchas resultantes da subdivisão de uma área representam pequenas amostras das manchas iniciais, pelo que é pouco provável que todas as espécies estejam representadas nessas manchas; b) com a diminuição do tamanho das manchas é comum a redução da diversidade de habitats e consequentemente do número e variedade de espécies que os ocupam; c) as áreas menores suportam populações menores, logo menor variabilidade específica (Albergaria, 2006). Assim, estudos sobre a Ecologia da Paisagem contribuíram para compreender os efeitos da fragmentação da paisagem sobre os fatores biofísicos. Adicionalmente, estas pesquisas podem também contribuir para o conhecimento das atividades socioeconómicas (Wickham et al., 2000). De facto, a paisagem e a sua consequente fragmentação é uma transição dinâmica e continua, não só resultado de processos naturais, como também, de alterações económicas e de valores culturais (Antrop \& Van Eetvelde, 2000).

\subsection{Determinação da heterogeneidade e estrutura espacial da paisagem - métricas da paisagem}

A determinação da heterogeneidade e estrutura espacial insere-se num campo disciplinar que propõe uma abordagem holística e de síntese ao estudo da diversidade e transformação da paisagem, a ecologia da paisagem.

Focando o estudo da interação entre padrões espaciais e processos ecológicos, isto é, as causas e consequências da heterogeneidade espacial em diferentes escalas (Turner et al., 
2001), a paisagem é tida como uma área heterogénea composta por um conjunto de ecossistemas que interagem entre si e que se repetem de forma similar no espaço ( $R$. Forman \& M. Godron, 1986), sendo que o resultado é mais do que a soma das suas partes (Antrop, 2000). Assim, a ecologia da paisagem envolve o estudo de padrões da paisagem, a interação entre manchas no interior do mosaico da paisagem, e a forma como padrões e interações mudam no tempo. Considera ainda o desenvolvimento e dinâmica da heterogeneidade espacial e os seus efeitos nos processos ecológicos (Couto, 2004; Laranjeira, 2012).

A relação entre a heterogeneidade e a fragmentação é bastante complexa. A extensão desta relação depende do grau de perturbação e dos fatores ambientais subjacentes (Antwi, Krawczynski, \& Wiegleb, 2008). A heterogeneidade espacial pode ser determinada pela diversidade de fatores ambientes, assim, o aumento da heterogeneidade acarreta um maior número de tipos de manchas o que implica, por exemplo, um aumento do número de nichos potencias e, consequentemente, um maior número de espécies que poderão coexistir. A heterogeneidade pode ainda atuar como um fator estabilizador (p.e. ao dispersar o risco). Portanto, a heterogeneidade da paisagem aumenta a qualidade da paisagem enquanto que a fragmentação promove o seu empobrecimento através da criação de atrito, isolamento e diminuição dos habitats (Andrén, 1994 upund Leitão \& Ahern, 2002). Segundo a ecologia da paisagem, uma paisagem estrutura-se por três tipos de elementos: manchas, corredores e matriz. Estes elementos são a raiz cognitiva que permite a comparação entre paisagens distintas, permitindo desenvolver princípios gerais (Casimiro, 2000). Para essa comparação são usadas métricas da paisagem que permitem a quantificação da estrutura da paisagem e a identificação de padrões e interações entre as manchas do mosaico da paisagem e possibilitam identificar como estas mudam ao longo do tempo. Enquanto manchas individuais possuem relativamente poucas características espaciais (tamanho, perímetro, forma), grupos de manchas já possuem um grupo mais alargado de características associativas, dependendo da forma de agrupamento - com base numa única classe (tipo de mancha) ou resultante da junção de múltiplas classes, podendo mesmo incluir toda a paisagem (McGarigal \& Marks, 1995). Assim, as métricas da paisagem podem ser calculadas para cada mancha (métricas de mancha), para todas as manchas de 
um dado tipo de mancha (métricas de classe) ou para a paisagem total (métricas de paisagem) (Couto, 2004; Griffith, Martinko, \& Price, 2000; McGarigal \& Marks, 1995).

As métricas da paisagem são algoritmos que quantificam características espaciais específicas de manchas, classes de manchas ou mosaico da paisagem (Couto, 2004). Estas métricas definem-se em duas categorias: as que quantificam a composição da paisagem sem referência aos atributos espaciais, ou as que quantificam a configuração espacial da paisagem, requerendo informação espacial para os seus cálculos (Gustafson, 1998). A composição, uma característica não explicitamente espacial, é facilmente quantificada e refere-se a características associadas à variedade e abundância de tipos de manchas no interior da paisagem (Botequilha Leitão \& Ahern, 2002; Couto, 2004). As principais medidas de composição são a proporção da paisagem em cada tipo de mancha, a riqueza, a uniformidade, o domínio e a diversidade dos tipos de manchas, exemplos disso são a área (McGarigal \& Marks, 1995) e o índice de Simpson's (Gustafson, 1998; McGarigal \& Marks, 1995). A configuração espacial das propriedades do sistema é mais difícil de quantificar e tem como objetivo a descrição das características espaciais de manchas individuais ou as relações espaciais entre múltiplas manchas (Botequilha Leitão \& Ahern, 2002; Couto, 2004). Os principais aspetos da configuração são o tamanho da mancha e densidade, a complexidade da forma da mancha, isolamento/proximidade, o contraste e o contágio e difusão, como por exemplo, a razão perímetro-área.

Assim, embora a literatura esteja repleta de métricas para descrever o padrão espacial, apenas existem duas componentes (composição e configuração) e poucos aspetos de cada uma delas. Ou seja, muitas dessas métricas medem aspetos similares do padrão da paisagem e estão correlacionadas entre si, porque existem poucas medidas primárias passíveis de serem extraídas (tipo de mancha, área, orla e tipo de vizinhança) o que as torna empiricamente redundantes (Couto, 2004; Cushman, McGarigal, \& Neel, 2008). O processo de seleção e avaliação das métricas a utilizar para descrever o padrão espacial da paisagem revela-se, portanto, fundamental e deve basear-se nos objetivos da pesquisa, nas características espaciais e nos processos ecológicos sob avaliação (Antrop \& Van Eetvelde, 2000; Bailey et al., 2007).

A heterogeneidade e a fragmentação da paisagem são processos e características mensuráveis. As métricas da paisagem tornam estes processos medíveis quantitativamente, 
promovendo um melhor entendimento dos processos e padrões da paisagem (Botequilha Leitão \& Ahern, 2002).

\section{Metodologia}

\subsection{Seleção da área de estudo}

Este estudo foi levado a cabo no âmbito de um projeto mais abrangente com vista à determinação da influência da fragmentação da floresta natural nas comunidades liquénicas. Assim, pretendíamos selecionar uma área de estudo que apresentasse homogeneidade florestal de modo a eliminar o maior número de variáveis não quantificáveis. Tendo em vista este objetivo, selecionamos a "sub-região homogénea" da Aboboreira proposta no Plano Regional de Ordenamento Florestal do Tâmega - PROF-T (PROF-T, 2007) (figura 1).

A Aboboreira é uma área montanhosa localizada no noroeste de Portugal que se reparte entre os Municípios de Amarante, Baião e Marco de Canaveses. Atinge aproximadamente os 1000m e tem uma orientação dominante de NE-SO. Geologicamente pertence à Zona Centro-Ibérica e é constituída essencialmente por granito e rochas metassedimentares (Honrado \& Vieira, 2009). A vegetação florestal típica consiste em florestas de carvalhos dominadas por Quercus robur (Alves, Honrado, Alves, Vieira, \& Séneca, 2007; Honrado \& Vieira, 2009). A Aboboreira apresenta características únicas em termos de biodiversidade que importa preservar sobretudo considerando que esta área é bastante assolada por fogos florestais, levando à fragmentação e constante perda de habitat natural.

Porque situado no extremo oriental do distrito do Porto, este território distingue-se pelas suas características de transição em termos naturais e níveis de desenvolvimento (Honrado \& Vieira, 2009).

Estudos anteriores (Honrado \& Vieira, 2009) revelam que os Municípios onde se insere a Aboboreira apresentam fenómenos de expansão urbana assentes numa estrutura de propriedade em minifúndio, sendo caracterizados por um povoamento disperso, mas sustentado em eixos, quer de linhas de água quer de rodovias. No entanto, nas freguesias 
mais serranas, caso da área em estudo, os aglomerados populacionais concentram-se nas zonas ribeirinhas ou a meia encosta em zonas de chãs.

O estudo socioeconómico dos Municípios onde a Aboboreira se enquadra permite perceber a sua evolução nos últimos 20 anos. A evolução populacional apresentou envelhecimento e decréscimo populacional (Tabela 1 do Anexo) (INE, 2011a; INE, 2011b).

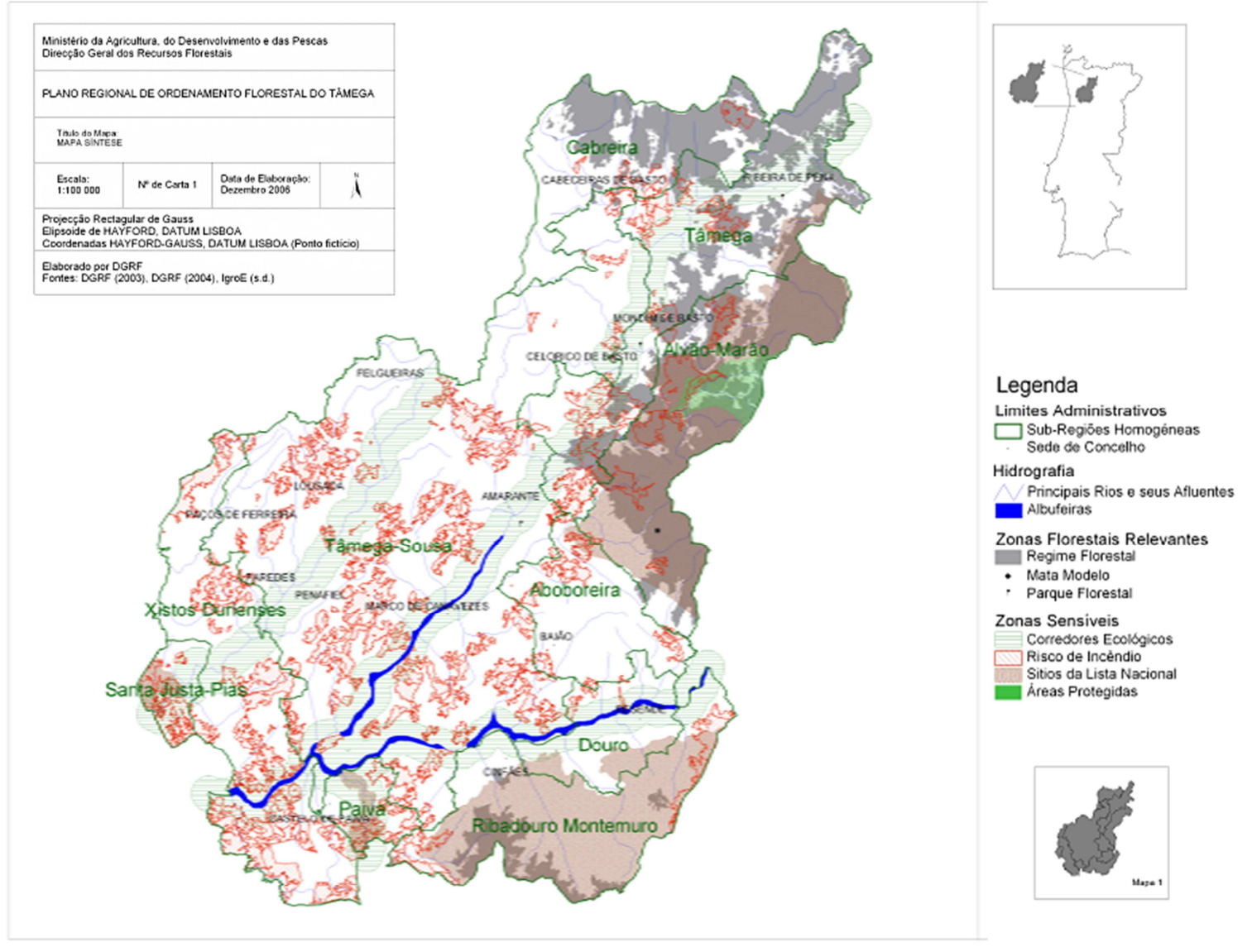

Figura 1 Mapa síntese. Modificado do PROF-T (2007).

\subsection{Determinação das métricas e evolução da paisagem}

Para avaliarmos a distribuição espacial da ocupação do solo e as áreas com grandes níveis de fragmentação da paisagem utilizou-se a extensão Patch Analyst do ArcGis (Elkie, Kaukinen, \& Carr, 2012) e a carta de ocupação do solo de 1990 (IGP). O primeiro passo metodológico constituiu na criação de uma malha regular de elementos hexagonais com uma área constante de 50 ha. Este processo possibilitou delinear uma rede de amostragem homogénea ao longo da área de estudo. Para cada um dos elementos da malha de 
amostragem foram calculadas as áreas com maior nível de fragmentação, para isso recorreu-se a métricas de área (CA), a métricas de densidade e tamanho (NumP e MPS) e a métricas de forma (MSI, MPAR e MPFD). Foram ainda produzidas estatísticas zonais dos dados categóricos de modo a resumir os atributos dos pixéis pertencentes a cada unidade hexagonal. Estas métricas permitiram obter um valor médio de classe de ocupação do solo por unidade hexagonal, assim como determinar a ocupação do solo mais frequente em cada hexágono.

Para a análise da evolução da paisagem foram utilizadas Cartas de Ocupação do Solo (COS) de Portugal Continental de diferentes anos (1990 e 2007) (IGP). Numa primeira fase procedeu-se à análise por data, seguida de sistematização e síntese dos valores relativos a cada classe de ocupação do solo, para posterior aplicação de matrizes de transição e de análise de sobreposição no que se refere ao estudo da evolução da ocupação do solo. Esta análise foi realizada com recurso ao programa Map Comparison Kit 3.2.2 (Visser \& de Nijs, 2006). Este programa possibilitou igualmente o cálculo de índices de estatística Kappa (Kappa, Fuzzy Kappa, KHisto e KLocation). Para a análise da evolução quantitativa da paisagem entre 1990 e 2007 recorreu-se ao programa FRAGSTATS 3.4 (McGarigal \& Marks, 1995). Aplicaram-se índices de diversidade (SIDI e SIEI), índices de área (LPI, AREA-MN, AREA-SD e AREA-CV) e índices de agregação (CONTAG, PD e NP) às cartas de ocupação do solo de 1990 e 2007, dados que foram posteriormente confrontados.

\section{Resultados}

\subsection{Distribuição espacial da ocupação do solo}

Os mapas resultantes da estratificação espacial e altimétrica permitiram deduzir as características marcadamente rurais deste território e assinalar a heterogeneidade da área de estudo. São, portanto, facilmente identificadas quatro grandes unidades de distribuição espacial (Figura 2), que vão de encontro a estudos anteriores realizados na Aboboreira por Honrado e Vieira (2009):

Áreas de vale - Áreas de menor altitude ao longo das linhas de água. Inclui áreas urbanas descontínuas e dispersas, numa matriz de áreas agrícolas anuais intercaladas 
com culturas permanentes. A floresta é composta por pequenas manchas de folhosas com resinosas.

Áreas de encosta - Áreas de altitudes intermédias, com ocupação agrícola e florestal associada a pequenos aglomerados urbanos dispersos. As classes de maior representação espacial são os incultos e as manchas florestais.

Áreas florestais de altitude - Áreas localizadas nos cumes das elevações, que possuem tipicamente uma matriz de incultos ou vegetação herbácea e arbustiva associada a espaços florestais dispersos ou degradados, onde surgem manchas de quercíneas e resinosas.

Áreas de montanha - Áreas de maior altitude, em relevo muito acidentado, em que as classes de ocupação mais frequentes são a de rocha nua ou vegetação esparsa, com áreas residuais de floresta.

As divisões acima indicadas são facilmente explicadas, uma vez que os espaços agrícolas predominam em áreas que apresentam uma maior amenidade climática, declives reduzidos e erosão baixa, e que as comunidades rurais localizam-se junto aos espaços agrícolas, formando aglomerados dispersos. Em termos gerais, o aumento da altitude correlaciona-se com o acréscimo dos declives, a diminuição da profundidade do solo e uma menor amenidade climática, resultando numa diminuição da densidade populacional e condicionando a implantação de atividades como a prática agrícola e florestal. 

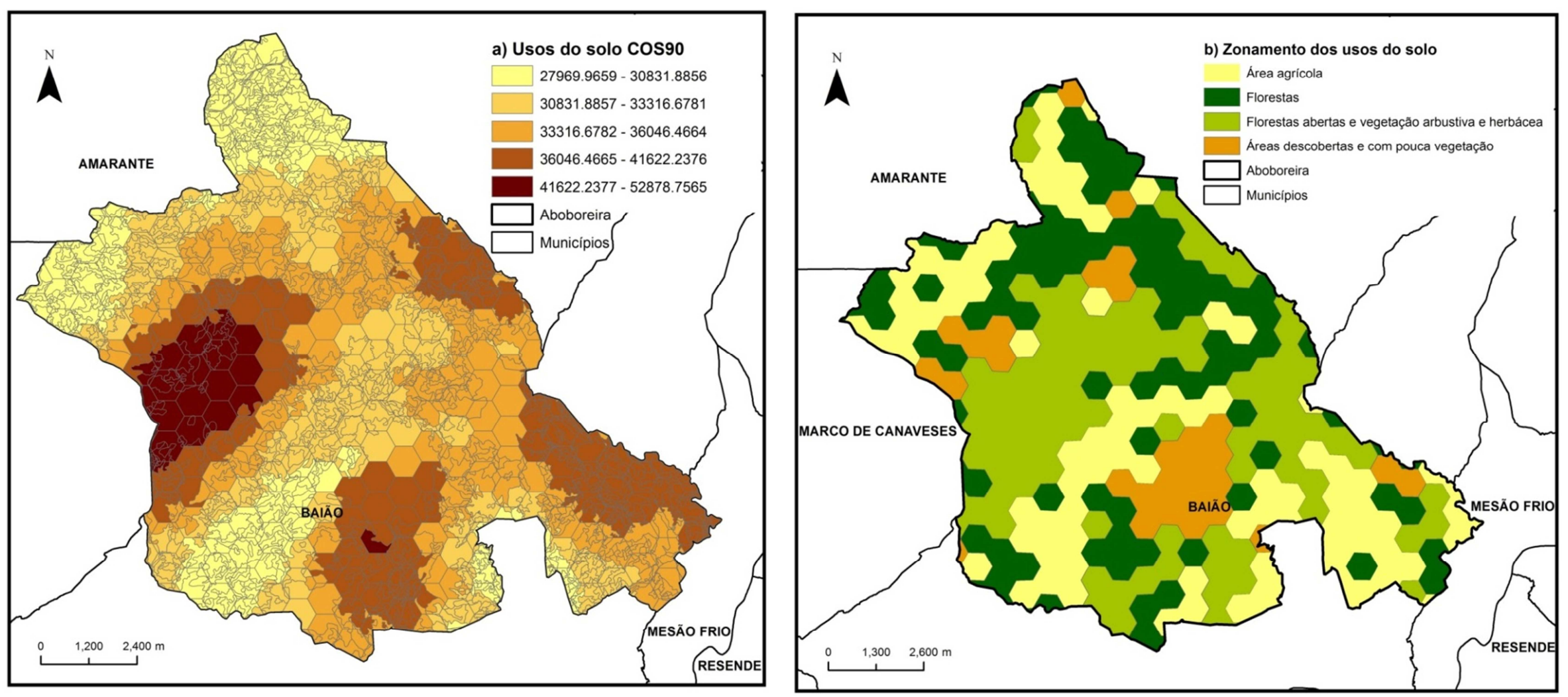

Figura 2 Zonamento regional a) Distribuição média da ocupação do solo b) Zonamento da ocupação do solo. Fonte: PROF-T e COS’90. 


\subsection{Determinação de áreas com grandes níveis de fragmentação da paisagem}

A análise das métricas de classe aplicadas a células hexagonais permitiu identificar áreas de maior fragmentação na paisagem em estudo (Figura 3).

O número de manchas (NumP) corresponde ao número máximo de classes individuais existentes em cada célula. É visível que, sobretudo em áreas bastante fragmentadas, as classes de ocupação do solo são variadas, existindo uma grande diversidade e intercalação entre si (Figura 3a).

A métrica MPS fornece a média do tamanho das manchas contidas em cada célula, sendo que quanto maior for a média, maior será a mancha ou menor será o número de manchas por hexágono. Esta medida simples permite ter uma perceção das áreas mais fragmentadas. Mais uma vez, é notória a menor fragmentação nos topos de montanha e uma crescente fragmentação em áreas de vale (Figura 3b).

Podemos verificar que as células com maior área (CA) se localizam nas cotas mais elevadas, onde geralmente se encontram solos de baixa produtividade quer agrícola quer florestal, mantendo-se extensas áreas inalteradas de matos e vegetação arbustiva (Figura $3 c$ ). As áreas de encosta e vale são altamente subdivididas e alternam entre povoamentos florestais e agrícolas, por vezes cortados por tecido urbano descontínuo.

O índice de forma médio (MSI) é igual a 1 quando todas as manchas são circulares e aumenta com a irregularidade da forma. Assim, podemos observar uma distribuição espacial, não uniforme, de altos valores de MSI (Figura 4a). Nota-se uma certa tendência para formas não complexas. Este resultado pode ser faccioso, uma vez que as células hexagonais cortam as manchas, e manchas menores tendem a ser menos irregulares.

A dimensão fractal média das manchas (MPFD) é também uma medida de complexidade da forma, e varia entre 1, para formas com perímetros simples, e 2, para formas mais complexas e indiciadoras de fragmentação da paisagem. Os valores próximos de 2 encontram-se na vizinhança de aglomerados populacionais, áreas agrícolas ou em áreas onde se procedeu ao cultivo intensivo de espécies exóticas de crescimento rápido (eucaliptos), enquanto que manchas com perímetros mais simples estão reduzidas a áreas de montanha (Figura 4c). 

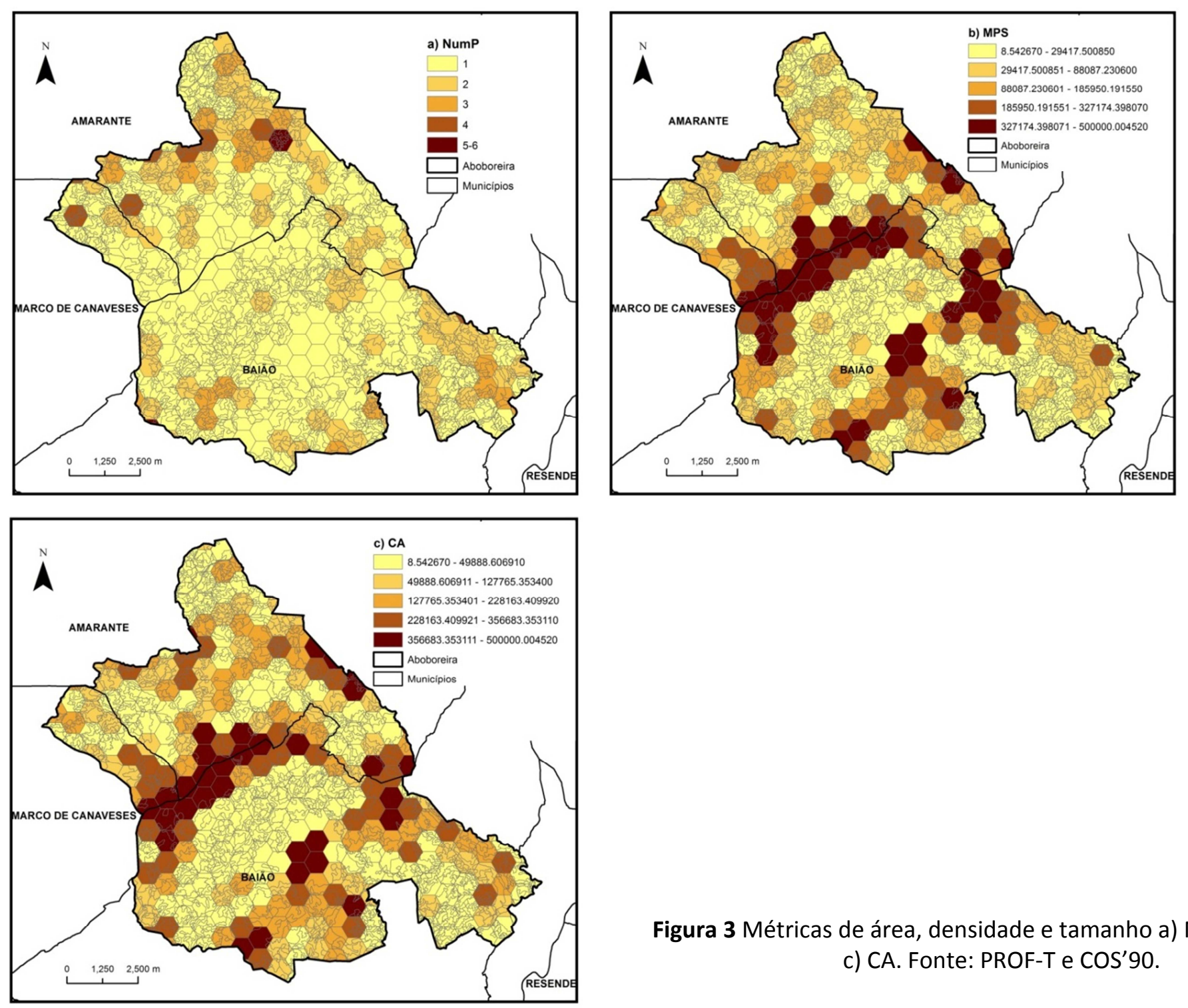

Figura 3 Métricas de área, densidade e tamanho a) NumP; b) MPS e c) CA. Fonte: PROF-T e $\operatorname{COS}^{\prime} 90$. 

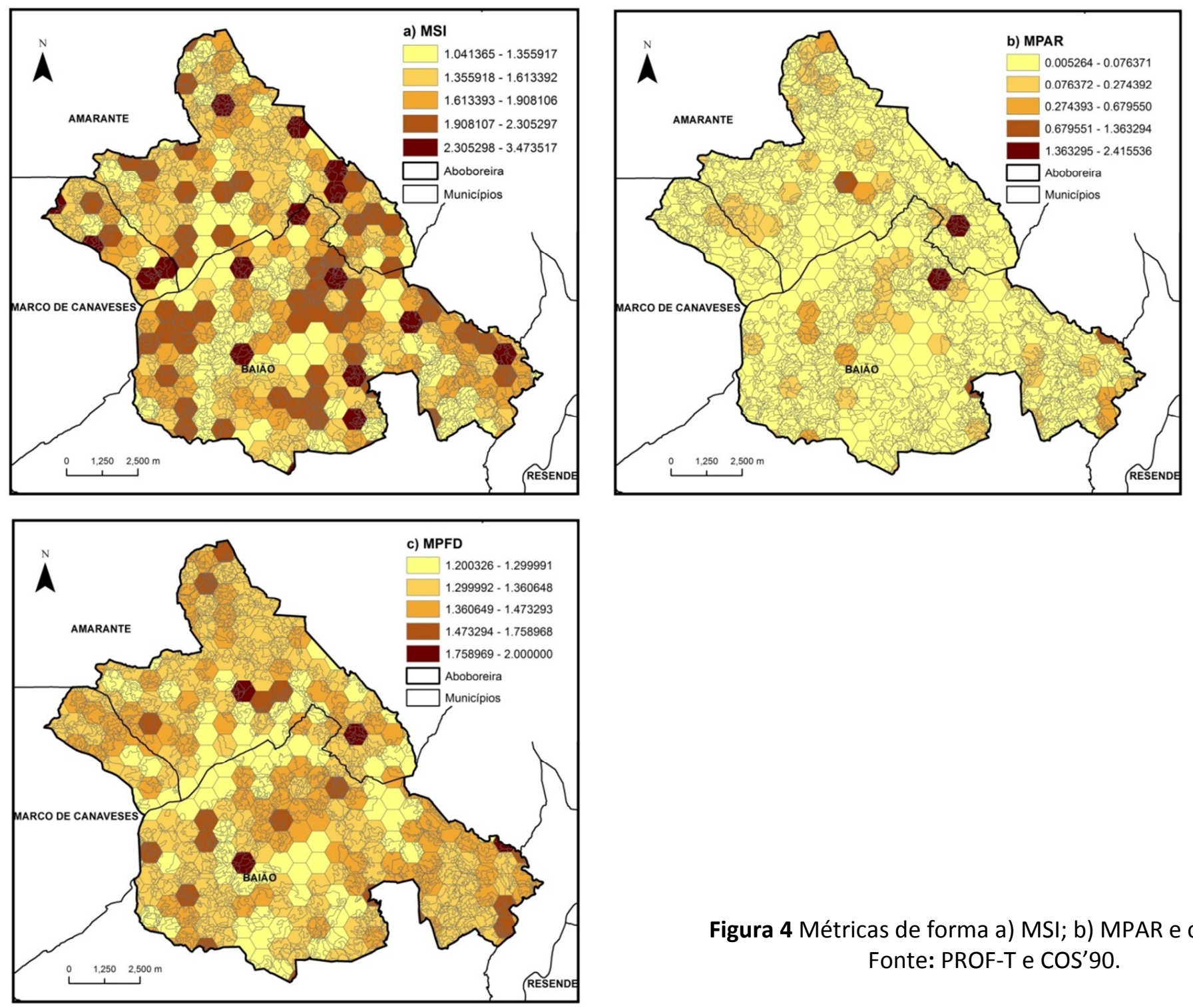

Figura 4 Métricas de forma a) MSI; b) MPAR e c) MPFD. Fonte: PROF-T e $\operatorname{COS}^{\prime} 90$. 
O MPAR, média da razão perímetro-área, mostra uma distribuição bastante uniforme dos valores, apresentando, no entanto, valores mais altos para as áreas com maior número de manchas (Figura 4b).

As métricas da paisagem aplicadas às unidades hexagonais permitiram determinar que a fragmentação da paisagem da Aboboreira é particularmente evidente em áreas de encosta e vale, e que nestas existe uma grande diversidade e intercalação de ocupações do solo. Por outro lado, nos topos das montanhas, persistem manchas pouco fragmentadas, com grandes áreas e pouca complexidade de forma.

\subsection{Evolução da paisagem}

Este subcapítulo aborda a evolução da ocupação do solo na Aboboreira. Para o estudo evolutivo da ocupação do solo aplicaram-se métricas da paisagem (Kappa, Fuzzy Kappa, KHisto e KLocation) às cartas de ocupação do solo de 1990 e 2007 (Figuras 5a e 5b). A estatística Kappa é uma medida de concordância e mede o grau de concordância além do que seria esperado somente pelo acaso (Yuan, Sawaya, Loeffelholz, \& Bauer, 2005), permitindo descrever se há ou não concordância entre dois ou mais fatores, neste caso o grau de concordância entre categorias de ocupação do solo de diferentes datas. Preferiu-se esta análise a uma análise simples de percentagem correta (o número de células iguais sobre o número total de células), porque esta muitas vezes não está de acordo com a análise visual dos mapas e, como demonstrado por Pontius (2002), mapas gerados ao acaso podem ter grande similaridade entre si, sobretudo quando possuem um pequeno número de categorias, o que se deve sobretudo ao facto de não ser considerada a configuração espacial dos pixéis (Mas, Pérez-Vega, \& Clarke, 2012).

O Kappa varia entre -1 e 1, o valor 1 indica total concordância e valores próximos de 0 representam nenhuma concordância. Um valor de Kappa menor que zero sugere que a concordância encontrada foi menor do que a esperada por acaso. Neste estudo o valor obtido para o Kappa é baixo (0.35) e revela portanto pouca concordância. Como se pode observar na Figura 5c, existem grandes diferenças entre os mapas referentes ao $\operatorname{COS}^{\prime} 90$ e $\operatorname{COS}^{\prime} 07$.

O KHisto permite determinar a similaridade de quantidade, enquanto, o KLocation permite determinar a similaridade de localização. Assim podemos verificar que a grande 
alteração na paisagem resultou de alterações na quantidade de cada classe de uso e ocupação do solo (KHisto de 0.47 ) enquanto que a sua localização se mantem aproximadamente constante (KLocation de 0.74). A fração correta é mais elevada que o Kappa e indica que $50 \%$ do mapa sofreu alterações (Figura 5c). O valor do Fuzzy Kappa em células contíguas permite avaliar a concordância tendo em conta a quantidade e a localização, pelo que as diferenças podem ser realçadas ou diluídas. Neste estudo, o valor de Fuzzy Kappa é bastante pequeno (-0.17999), e aponta para diferenças significativas entre os dois mapas (Figura $5 d$ ).

O método de comparação do Kappa por categoria executa uma comparação célula a célula em relação a uma ocupação do solo específico. Este método fornece simultaneamente informação sobre as ocorrências nos dois mapas. No período considerado (1990 a 2007) verificaram-se fortes mudanças nas diferentes classes de ocupação do solo. Em termos gerais, por análise das matrizes de transição, verifica-se que a expansão dos territórios artificializados foi acompanhada por uma diminuição das áreas agrícolas e agroflorestais, mas acima de tudo, pela transição de áreas de culturas temporárias para tecido urbano (Figura 6, Tabelas 2 e 3 do Anexo).

O grande aumento (22.62\%) das florestas abertas e vegetação arbustiva e herbácea foi acompanhado pela diminuição das culturas permanentes, áreas agrícolas heterogéneas e florestas (Tabelas 2 e 3 do Anexo). Isto pode ser resultado da recuperação dos espaços agrícolas abandonados por vegetação autóctone e por perda do espaço florestal por devastação ou incêndio florestal. Simultaneamente, verifica-se uma expansão das áreas urbanas (1.79\%) em torno e no interior das áreas de vale a partir dos povoamentos já existentes (Figura 6). O crescimento urbano é acompanhado por um aumento significativo das áreas artificiais associadas, em particular áreas industriais, de comércio e transportes.

O menor valor de Kappa corresponde às áreas de extração de inertes (-0.00019), sendo este valor o resultado de perda de células e não de alteração de localização. A classe tecido urbano apresenta o maior valor de Kappa, bem como um dos valores mais altos de KHisto e KLocation, revelando uma menor alteração tanto em localização como em número de células (Figuras 6). 

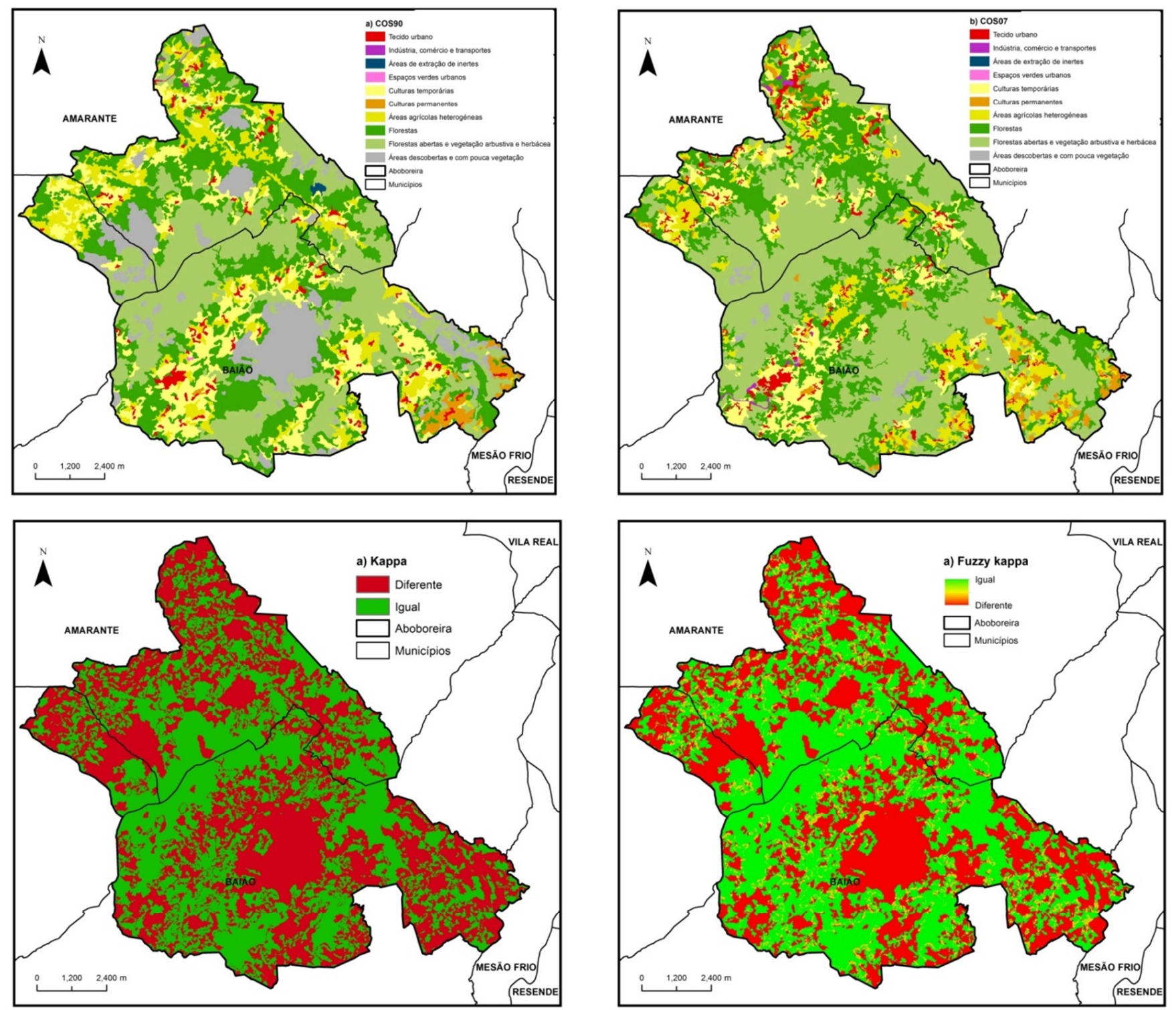

Figura 5 Mapas do nível II da carta de ocupação do solo de a) 1990 e b) 2007. Mapas resultantes da comparação entre os $\operatorname{COS}^{\prime} 90$ e $\operatorname{COS}^{\prime} 07$ para os índices c) Kappa e d) Fuzzy Kappa. Fonte: PROF-T, $\operatorname{COS}^{\prime} 90$ e COS'07. 

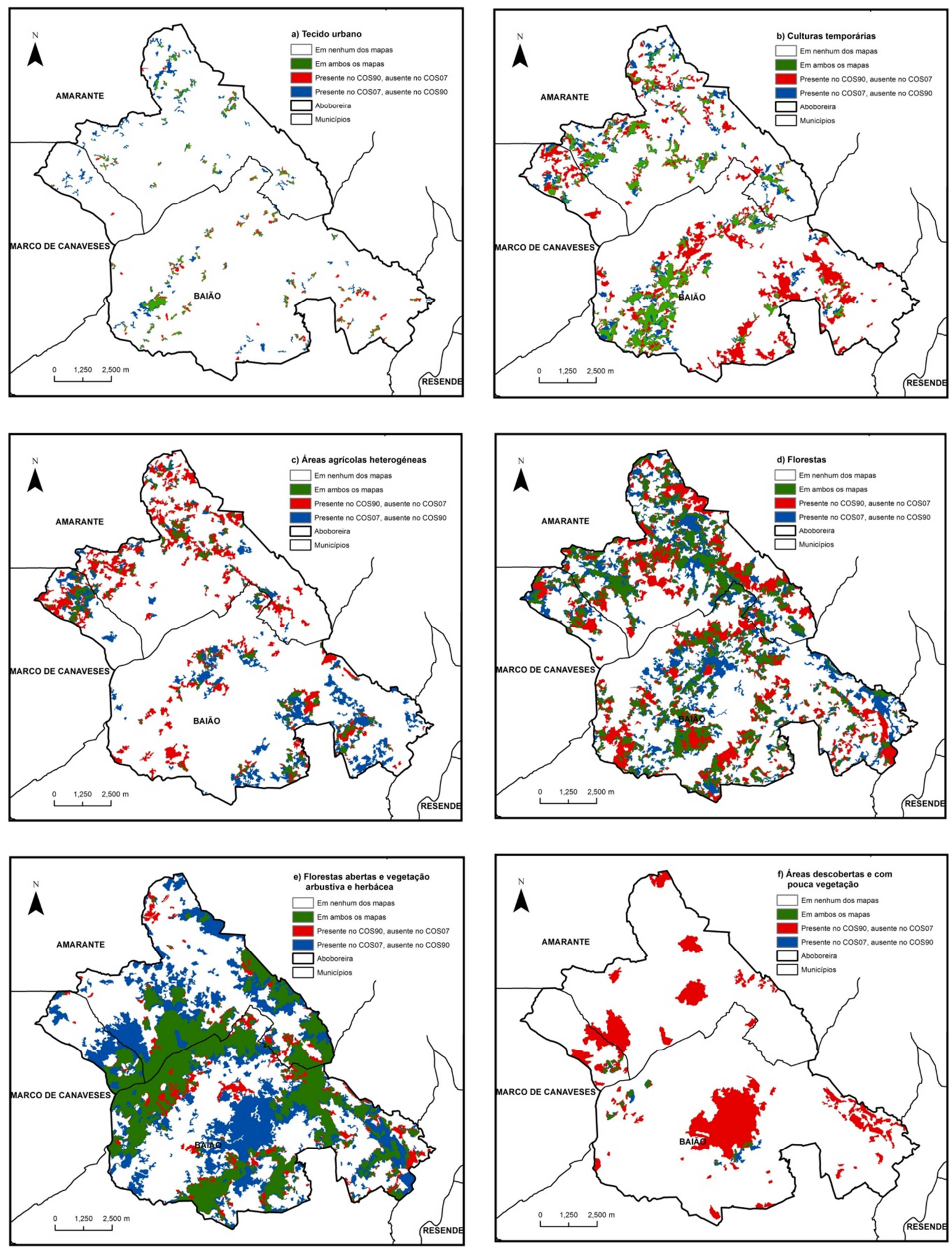

Figura 6 Mapas comparativos por categoria, a) Tecido urbano, b) Culturas temporárias, c) Áreas agrícolas heterogéneas, d) Florestas, e) Florestas abertas e vegetação arbustiva e herbácea e F) Áreas descobertas e com pouca vegetação.

Fonte: PROF-T, $\operatorname{COS}^{\prime} 90$ e $\operatorname{COS}^{\prime} 07$. 


\subsection{Evolução quantitativa da paisagem}

Neste subcapítulo aborda-se a evolução quantitativa da paisagem da Aboboreira entre os anos de 1990 e 2007.

O índice de riqueza das manchas (PR) é igual ao número de tipos de manchas presentes na paisagem e é a base para o cálculo dos índices de Shannon e Simpson (De Clercq et al., 2006), no nosso estudo revela que o número de tipos de ocupação do solo (classes) não sofreu alteração (Tabela 1).

Tabela 1 Resultados da análise das métricas da paisagem para os anos de 1990 e 2007 . Fonte: COS'90 e COS'07.

\begin{tabular}{lrr|lrr}
\hline & COS'90 $^{\prime}$ & COS'07 $^{\prime}$ & & COS'90 & COS'07 \\
\hline PR & 10 & 10 & PD & 5.7695 & 7.0684 \\
SIDI & 0.7802 & 0.6817 & LPI & 12.4926 & 19.0456 \\
SIEI & 0.8668 & 0.7574 & AREA-MN & 17.3327 & 14.1475 \\
CONTAG & 56.6972 & 61.605 & AREA-SD & 79.9094 & 120.4112 \\
NP & 755 & 925 & AREA-CV & 461.0337 & 851.1102 \\
\hline
\end{tabular}

O índice de diversidade de Simpson's (SIDI) reflete sobretudo as diferenças na riqueza das manchas e classifica-a ao longo de um gradiente, demonstrando neste caso, uma diminuição de diversidade entre 1990 e 2007. Em 1990 o SIDI revela que existe uma de probabilidade $78 \%$ de duas manchas escolhidas ao acaso serem de diferentes tipos de ocupação do solo, valor que diminui para 68\% em 2007.

Dos índices de uniformidade disponíveis no FRAGSTAT foi selecionado o índice de uniformidade de Simpson's (SIEI), porque é menos influenciado pelos usos de solo raros. Tendo por base este índice, o ano de 1990 apresentou maior uniformidade de ocupação do solo; a distribuição de área por tipo de ocupação do solo é de $76 \%$ da máxima uniformidade para o ano 2007 e de $87 \%$ para o ano de 1990.

O índice de contágio (CONTAG) faz uma análise célula a célula, medindo tanto a interdispersão de tipos de manchas (mistura de manchas de diferentes classes) como a dispersão de manchas (distribuição espacial de uma classe de manchas). Valores altos indiciam paisagens com manchas pouco numerosas, de grandes dimensões e próximas entre si, enquanto valores baixos indiciam paisagens com muitas unidades dispersas 
(Casimiro, 2000; O'Neill et al., 1988). O valor de CONTAG aproxima-se de 0 quando a distribuição entre tipos de manchas da mesma classe se torna menos equilibrada e de 100 quando todos os tipos de manchas estão igualmente adjacentes a todas as outras classes. O valor do índice é superior para 2007, o que reflete grandes manchas, menor densidade de manchas, mais contíguas, mais contínuas, menos diversidade na vizinhança e maior agregação de células (Tabela 1).

O número de manchas (NP) e a densidade da mancha (PD) são boas métricas para representar o gradiente de diversidade e heterogeneidade da paisagem dos diferentes anos. Estes índices indicam que o ano de 2007 apresenta um "grão mais fino", isto é, maior número de manchas, logo maior densidade, demonstrando uma maior heterogeneidade espacial. Neste estudo o NP e o CONTAG forneceram informações contrárias, indicando o NP uma maior heterogeneidade espacial para o ano de 2007 e o CONTAG para o ano de 1990. Estes dados são facilmente explicados se se tiver em consideração que o ano de 2007 apresenta manchas que sofreram regeneração por parte de espécies autóctones, e por conseguinte integram as áreas anteriormente degradadas, agrupando-se a estas e aumentando o número de pixéis com o mesmo valor, e dando portanto a ilusão de maior homogeneidade. O índice da mancha mais larga (LPI) é obtido através da divisão da área da maior mancha pela área total da paisagem e é expressa em percentagens (McAlpine and Eyre, 2002). O LPI indica que cerca de $19 \%$ da paisagem de 2007 é preenchida por uma única mancha, apesar de ser o ano que demonstra também maior heterogeneidade espacial. Este índice não é um bom indicador de heterogeneidade, já que a maior mancha pode corresponder a uma classe com pouco número de manchas. A sua grande importância prende-se, por exemplo, com a necessidade por parte de algumas espécies de uma determinada dimensão da mancha como área vital (Casimiro, 2000) ou quando se está a calcular a resistência da paisagem à fragmentação (De Clercq et al., 2006). O tamanho das manchas é em média maior (AREA-MN) em 1990. A dimensão média das manchas é um bom indicador da fragmentação de habitats e é tanto mais significativa quanto maior o número de manchas ou a dimensão total da paisagem. O desvio padrão do tamanho das manchas (AREA-SD) parece indicar uma maior homogeneidade geral em 1990. Sendo uma medida de dispersão do tamanho das manchas, este índice dá 
indicações quanto ao padrão de uniformidade do tamanho das manchas, o que permite determinar com maior acuidade a heterogeneidade da paisagem (Casimiro, 2000). A configuração da paisagem em 2007 evidencia um maior número de manchas, geralmente mais pequenas, uma maior densidade e um coeficiente de variação da sua dimensão de maior valor (AREA-CV), pelo que é facilmente deduzida a maior heterogeneidade da paisagem comparativamente a 1990.

Em resumo, os índices de diversidade (SIDI e SIEI) indicam diminuição de diversidade e uniformidade de ocupação do solo, os índices de área (LPI, AREA-MN, AREA-SD e AREA-CV) apontam para maior heterogeneidade da paisagem em 2007 e os índices de agregação (CONTAG, PD e NP) indicam que o ano de 1990 apresenta menor heterogeneidade espacial. A conjugação destes índices revela um aumento da fragmentação da paisagem da sub-região homogénea da Aboboreira, através do aumento do número de manchas, diminuição do tamanho das mesmas e aumento da dispersão entre elas.

\section{Discussão e conclusão}

Nos últimos anos têm-se verificado grandes alterações na ocupação do solo da Serra da Aboboreira. Estas alterações foram sobretudo devidas às dinâmicas relacionadas com as atividades e usos associados à ação humana, que conjuntamente com as condições ambientais existentes condicionaram a ocupação do solo. Salientam-se a disseminação pelo território de povoamentos florestais de pinheiro bravo e eucalipto, a considerável recolonização florestal resultante do abandono dos terrenos agrícolas de altitude, menos apetecíveis do ponto de vista da prática agrícola atual, e uma grande expansão das áreas urbanas. Em conjunto acarretam perda de biodiversidade dos ecossistemas, diminuição da qualidade paisagística, menor número de locais de refúgio para a fauna selvagem e o aumento da vulnerabilidade a certos riscos, tais como, incidência de fogo florestais, cheias e aridez. A Aboboreira tornou-se significativamente mais urbana, menos agrícola e mais florestal. Estes resultados confirmam as tendências assinaladas noutros estudos sobre paisagens mediterranicas de montanha portuguesas (Viana \& Aranha 2008) e europeias (Pelorosso, Leone, \& 
Boccia, 2009). No entanto, algumas paisagens de montanha mediterrânicas mostram uma tendência para a substituição das culturas anuais por prados, tornando-se mais monotonas (Pôças et al, 2011). Esta diferença foi explicada por Pôças et al (2011) como resultado da aplicação de políticas agricolas regionais ou locais diferenciadas.

As alterações na ocupação do solo foram acompanhadas por uma generalizada tendência de aumento da fragmentação da paisagem da sub-região homogénea da Aboboreira, patente no aumento do número de manchas, na diminuição do tamanho das mesmas e no aumento da sua dispersão. Esta tendência de fragmentação foi particularmente evidente nas áreas de vale, que pela sua maior aptidão agrícola e atratividade para a ocupação humana, registaram um aumento significativo do número de manchas e uma diminuição generalizada da área das manchas.

A evolução e transformação da paisagem da Aboboreira enquadra-se, em suma, numa dinâmica mais geral que Geri et al. (2010) sintetizam em duas principais tendências: i) a expansão das manchas florestais em áreas de montanha devido ao abandono das áreas agrícolas; ii) um processo genérico de fragmentação e expansão das áreas agrícolas e urbanas, devido ao desenvolvimento de uma economia industrial.

Em conclusão os nossos resultados indicam que a Aboboreira está num processo ativo de fragmentação com consequente perda de habitats. Possuindo a Aboboreira manchas florestais importantes para a conservação da natureza e da biodiversidade, este estudo contribui para evidenciar a urgência de medidas de planeamento territorial que possam integrar a conservação de habitats com espécies em declínio em Portugal e a expansão das áreas urbanas que sustentam o desenvolvimento regional. Algumas medidas poderiam passar por incentivar a conversão de monoculturas florestais em floresta autóctone, aumentando a diversidade e a capacidade reguladora da paisagem; pela arborização dos terrenos agrícolas abandonados, fomentando o alargamento das áreas florestais; pela promoção e valorização dos seus produtos tradicionais (castanha, mel, plantas aromáticas, cogumelos) promovendo a diferenciação do território e dos seus produtos; pela gestão dos bosques ribeirinhos por forma a potenciar a vegetação arbustiva e arbórea nativa, pela promoção da conectividade através da criação de corredores ecológicos; pela monitorização centrada em aspetos de conservação e biodiversidade; e pela minimização do 
abandono da agricultura através de programas de desenvolvimento rural, evitando a desqualificação social, económica e ambiental do território.

Uma das propostas finais e que poderá facilitar a implementação das medidas anteriormente descritas é a de criação de uma Paisagem Protegida de Âmbito Regional. Uma proposta semelhante foi apresentada na Assembleia da República em 8 de Março de 2000 (Projeto de Lei n. 138/VIII). De acordo com esta proposta estão também Honrado \& Vieira (2009) que a descrevem como uma resposta cabal e direta ao anseio local de valorização destes espaços na perspetiva da valorização territorial.

\section{Referências}

Albergaria, C. (2006). Um olhar crítico sobre o conceito e a prática da Reserva Ecológica Nacional. Mestrado, Faculdade de Engenharia, Universidade do Porto, Porto. Retrieved from http://www.estig.ipbeja.pt/ ac_direito/Albergaria.pdf

Alves, H., Honrado, J., Alves, P., Vieira, C., \& Séneca, A. (2007). A flora e a vegetação da Serra da Aboboreira. In C. M. Amarante (Ed.), Serra da Aboboreira: a Terra, o Homem e os Lobos.

Andrén, H. (1994). Effects of habitat fragmentation on birds and mammals in landscapes with different proportions of suitable habitat: a review. Oikos, 71, 355-366.

Antrop, M. (2000). Background concepts for integrated landscape analysis. Agriculture, Ecosystems \& Environment, 77(1-2), 17-28. doi: http://dx.doi.org/10.1016/S0167-8809(99)00089-4

Antrop, M., \& Van Eetvelde, V. (2000). Holistic aspects of suburban landscapes: visual image interpretation and landscape metrics. Landscape and Urban Planning, 50(1-3), 43-58. doi: http://dx.doi.org/10.1016/S0169-2046(00)00079-7

Antwi, E. K., Krawczynski, R., \& Wiegleb, G. (2008). Detecting the effect of disturbance on habitat diversity and land cover change in a post-mining area using GIS. Landscape and Urban Planning, 87(1), 22-32. doi: http://dx.doi.org/10.1016/j.landurbplan.2008.03.009

Belinchón, R., Martínez, I., Escudero, A., Aragón, G., Valladares, F. (2007). Edge effects on epiphytic communities in a Mediterranean Quercus pyrenaica forest. Journal of Vegetation Science 18, 81-90.

Botequilha Leitão, A., \& Ahern, J. (2002). Applying landscape ecological concepts and metrics in sustainable landscape planning. Landscape and Urban Planning, 59(2), 65-93. doi: http://dx.doi.org/10.1016/S0169-2046(02)00005-1

Casimiro, P. (2000). Uso do Solo - Ecologia da Paisagem: Perspectivas de uma Nova Abordagem do Estudo da Paisagem em Geografia. Revista Geolnova, 2, 45-66.

Chen, T.-S., \& Lin, H.-J. (2013). Development of a framework for landscape assessment of Taiwanese wetlands. Ecological Indicators, 25(0), 121-132. doi: http://dx.doi.org/10.1016/j.ecolind.2012.09.012

Couto, P. (2004). Análise factorial aplicada a métricas da paisagem definidas em FRAGSTATS. Investigação Operacional, 24, 109-137

Cushman, S. A., McGarigal, K., \& Neel, M. C. (2008). Parsimony in landscape metrics: Strength, universality, and consistency. Ecological Indicators, 8(5), 691-703. doi: http://dx.doi.org/10.1016/j.ecolind.2007.12.002

De Clercq, E. M., Vandemoortele, F., \& De Wulf, R. R. (2006). A method for the selection of relevant pattern indices for monitoring of spatial forest cover pattern at a regional scale. International Journal of 
Applied Earth Observation and Geoinformation, 8(2), 113-125. doi: http://dx.doi.org/10.1016/j.jag.2005.07.002

Fahrig, L. (2003). Effects of habitat fragmentation on biodiversity. Annual Review of Ecology, Evolution, and Systematics, 34, 487-515.

Forman, R., \& Godron, M. (1986). Landscape Ecology. New York: John Wiley and Sons.

Geri, F., Amici, V., \& Rocchini, D. (2010). Human activity impact on the heterogeneity of a Mediterranean landscape. Applied geography, 30(3), 370-379.

Griffith, J. A., Martinko, E. A., \& Price, K. P. (2000). Landscape structure analysis of Kansas at three scales. Landscape and Urban Planning, 52(1), 45-61. doi: http://dx.doi.org/10.1016/S01692046(00)00112-2

Gustafson, E. (1998). Quantifying landscape spatial pattern: What is the state of the art. Ecosystems, 143-156

Honrado, J., \& Vieira, C. (2009). O património natural como factor de desenvolvimento e competitividade territoriais no Baixo Tâmega. O Presente e o Futuro do Património Natural dos concelhos de Amarante, Baião e Marco de Canaveses. Porto.

Hunter, M. (1996). Fundamentals of Conservation Biology. USA: Blackwell Science.

INE (2011a). Estimativas Anuais da População Residente. Instituto Nacional de Estatística Portugal, Lisboa.

INE (2011b). Indicadores Demográficos. Instituto Nacional de Estatística Portugal, Lisboa.

IUCN. (1980). World conservation strategy. Living resource conservation for sustainable development. Retrieved from http://data.iucn.org/dbtw-wpd/edocs/WCS-004.pdf

Johansson, P. (2008). Consequences of disturbance on epiphytic lichens in boreal and near boreal forests. Biological Conservation, 141(8), 1933-1944. doi: 10.1016/j.biocon.2008.05.013

Laranjeira, M. (2012). Estrutura Espacial e Processos Ecológicos: O estudo da Fragmentação dos Habitats. Revista de Geografia e Ordenamento do Território, n.o 1 (Junho). Centro de Estudos de Geografia e Ordenamento do Território. Pág. 59-83

Leitão, A., \& Ahern, J. (2002). Applying landscape ecological concepts and metrics in sustainable landscape planning. Landscape and Urban Planning, 59, 65-93.

Mas, J.-F., Pérez-Vega, A., \& Clarke, K. (2012). Assessing simulated land use/cover maps using similarity and fragmentation indices. Ecological Complexity, 11(0), 38-45. doi: http://dx.doi.org/10.1016/j.ecocom.2012.01.004

McAlpine, C., Eyre, T. (2002). Testing landscape metrics as indicators of habitat loss and fragmentation in continuous eucalypt forests (QId, Australia). Landscape Ecol. 17, 711-728.

McGarigal, K., \& Marks, B. (1995). FRAGSTATS: spatial pattern analysis program for quantifying landscape structure. Pacific Northwest Research Station, Portland, OR: USDA Forest Service.

Olff, H., \& Ritchie, M. E. (2002). Fragmented nature: consequences for biodiversity. Landscape and Urban Planning, 58(2-4), 83-92. doi: http://dx.doi.org/10.1016/S0169-2046(01)00211-0

O'Neill, R., Krummel, J., Gardner, R., Sugihara, G., Turner, M., Graham, R. (1988). Indices of landscape pattern. Landscape Ecol., 1, 153-162.

Pelorosso, R., Leone, A., \& Boccia, L. (2009). Land cover and land use change in the Italian central Apennines: A comparison of assessment methods. Applied Geography, 29(1), 35-48. doi: http://dx.doi.org/10.1016/j.apgeog.2008.07.003

Pôças, I., Cunha, M., Marcal, A., \& Pereira, L. S. (2011). An evaluation of changes in a mountainous rural landscape of Northeast Portugal using remotely sensed data. Landscape and Urban Planning, 101(3), 253-261. doi: http://dx.doi.org/10.1016/j.landurbplan.2011.02.030

Pontius, R. (2000). Quantification error versus location error in comparison of categorical maps. Photogrammetric Engineering and Remote Sensing 66 (8), 1011-1016. 
Pontius, R.(2002). Statistical methods to partition effects of quantity and location during comparison of categorical maps at multiple resolutions. Photogrammetric Engineering and Remote Sensing 68 (10), 1041-1049.

PROF-T. (2007). Plano Regional de Ordenamento Florestal do Tâmega. Lisboa: Comissão de acompanhamento.

Romero-Calcerrada, R., \& Perry, G. L. W. (2004). The role of land abandonment in landscape dynamics in the SPA 'Encinares del río Alberche y Cofio, Central Spain, 1984-1999. Landscape and Urban Planning, 66(4), 217-232. doi: http://dx.doi.org/10.1016/S0169-2046(03)00112-9

Turner, M., Gardner R., O'Neill R. (2001). Landscape Ecology in Theory and Practice: Pattern and Process. Springer-Verlag, New York, NY, USA.

Viana, H., Aranha, J. (2008). Estudo da alteração da cobertura do solo no Parque Nacional da Peneda Gerês (1995 e 2007). Análise temporal dos padrões espaciais e avaliação quantitativa da estrutura da paisagem. X Encontro de Utilizadores de Sistemas de Informação Geográfica, 14 a 16 de Maio de 2008 Oeiras.

Visser, H., \& de Nijs, T. (2006). The Map Comparison Kit. Environmental Modelling \&amp; Software, 21(3), 346-358. doi: 10.1016/j.envsoft.2004.11.013

Wiens, J. (1976). Population responses to patchy environments. Ann. Rev. Ecol. Syst., 7.

Wickham, J. D., O’Neill, R. V., Jones, K. B. (2000). Forest fragmentation as an economic indicator. Landsc. Ecol. 15, 171-179.

Yuan, F., Sawaya, K. E., Loeffelholz, B. C., \& Bauer, M. E. (2005). Land cover classification and change analysis of the Twin Cities (Minnesota) Metropolitan Area by multitemporal Landsat remote sensing. Remote Sensing of Environment, 98(2-3), 317-328. doi: http://dx.doi.org/10.1016/j.rse.2005.08.006 


\section{ANEXO}

Tabela 1 Indicadores demográficos, taxa de crescimento efetivo e variação populacional e índice de envelhecimento. Fonte: INE,2011a; INE, 2011b.

\begin{tabular}{|c|c|c|c|c|}
\hline Local de residência & $\begin{array}{l}\text { Período de } \\
\text { referência dos } \\
\text { dados }\end{array}$ & $\begin{array}{l}\text { Taxa de crescimento } \\
\text { efetivo (\%) por Local } \\
\text { de residência; Anual }\end{array}$ & $\begin{array}{c}\text { Índice de } \\
\text { envelhecimento (N..) } \\
\text { por Local de } \\
\text { residência; Anual }\end{array}$ & $\begin{array}{l}\text { Variação populacional } \\
\text { (N.o) por Local de } \\
\text { residência; Anual }\end{array}$ \\
\hline & 2011 & -0.38 & 100.3 & -215 \\
\hline \multirow[t]{3}{*}{ Amarante } & 2001 & 0.02 & 64.4 & 13 \\
\hline & 1991 & $x$ & 44.2 & -33 \\
\hline & 2011 & -0.82 & 129.9 & -167 \\
\hline \multirow[t]{3}{*}{ Baião } & 2001 & -0.58 & 89.5 & -129 \\
\hline & 1991 & $x$ & 65.9 & -53 \\
\hline & 2011 & 0.03 & 72.7 & 14 \\
\hline \multirow[t]{2}{*}{ Marco de Canaveses } & 2001 & 0.67 & 52.7 & 352 \\
\hline & 1991 & $x$ & 41 & 125 \\
\hline
\end{tabular}

Tabela 2 Tabela de alterações de ocupação do solo por classes. Fonte $\operatorname{COS}^{\prime} 90$ e $\operatorname{COS}^{\prime} 07$.

\begin{tabular}{|c|c|c|c|c|c|c|}
\hline Classe & Persistência & Perdas & Ganhos & $\begin{array}{c}\text { Persistência } \\
\text { (\%) }\end{array}$ & $\begin{array}{c}\text { Perdas } \\
\text { (\%) }\end{array}$ & $\begin{array}{c}\text { Ganhos } \\
(\%)\end{array}$ \\
\hline Tecido urbano & 3977 & 2818 & 5844 & 1.22 & 0.86 & 1.79 \\
\hline Indústria, comércio e transportes & 30 & 257 & 796 & 0.01 & 0.08 & 0.24 \\
\hline Áreas de extração de inertes & 0 & 368 & 33 & 0 & 0.11 & 0.01 \\
\hline Espaços verdes urbanos & 20 & 39 & 10 & 0.01 & 0.01 & 0 \\
\hline Territórios artificializados & 4027 & 3482 & 6683 & 1.23 & 1.06 & 2.04 \\
\hline Culturas temporárias & 18891 & 30534 & 11890 & 5.77 & 9.33 & 3.63 \\
\hline Culturas permanentes & 1591 & 3587 & 6314 & 0.49 & 1.1 & 1.93 \\
\hline Áreas agrícolas heterogéneas & 9717 & 26381 & 21006 & 2.97 & 8.06 & 6.42 \\
\hline Áreas agrícolas e agro-florestais & 30199 & 60502 & 39210 & 9.23 & 18.49 & 11.99 \\
\hline Florestas & 50313 & 44838 & 39712 & 15.38 & 13.71 & 12.14 \\
\hline Florestas abertas e vegetação arbustiva e herbácea & 80640 & 15473 & 73988 & 24.65 & 4.73 & 22.62 \\
\hline Áreas descobertas e com pouca vegetação & 1350 & 36330 & 1032 & 0.41 & 11.1 & 0.32 \\
\hline Florestas e meios naturais e semi-naturais & 132303 & 96641 & 114732 & 40.44 & 29.54 & 35.07 \\
\hline Total & 166529 & 160625 & 160625 & 50.9 & 49.1 & 49.1 \\
\hline
\end{tabular}


Tabela 3 Matriz de transição de usos do solo na Aboboreira. Fonte: $\operatorname{COS}^{\prime} 90$ - $\operatorname{COS}^{\prime} 07$.

\begin{tabular}{|c|c|c|c|c|c|c|c|c|c|c|c|}
\hline $\cos 90 \backslash \cos 07$ & $\begin{array}{l}\text { Tecido } \\
\text { urbano }\end{array}$ & $\begin{array}{l}\text { Indústria, } \\
\text { comércio e } \\
\text { transportes }\end{array}$ & $\begin{array}{c}\text { Áreas de } \\
\text { extração de } \\
\text { inertes }\end{array}$ & $\begin{array}{l}\text { Espaços } \\
\text { verdes } \\
\text { urbanos }\end{array}$ & $\begin{array}{c}\text { Culturas } \\
\text { temporárias }\end{array}$ & $\begin{array}{c}\text { Culturas } \\
\text { permanentes }\end{array}$ & $\begin{array}{c}\text { Áreas agrícolas } \\
\text { heterogéneas }\end{array}$ & Florestas & $\begin{array}{c}\text { Florestas } \\
\text { abertas e } \\
\text { vegetação } \\
\text { arbustiva e } \\
\text { herbácea }\end{array}$ & $\begin{array}{c}\text { Áreas } \\
\text { descobertas e } \\
\text { com pouca } \\
\text { vegetação }\end{array}$ & $\begin{array}{l}\text { Total } \\
\text { cos90 }\end{array}$ \\
\hline Tecido urbano & 3977 & 73 & 0 & 0 & 1009 & 151 & 1121 & 282 & 173 & 9 & 6795 \\
\hline $\begin{array}{l}\text { Indústria, comércio e } \\
\text { transportes }\end{array}$ & 43 & 30 & 0 & 0 & 25 & 0 & 20 & 158 & 11 & 0 & 287 \\
\hline Áreas de extração de inertes & 0 & 0 & 0 & 0 & 0 & 0 & 0 & 192 & 176 & 0 & 368 \\
\hline Espaços verdes urbanos & 22 & 14 & 0 & 20 & 0 & 0 & 0 & 1 & 2 & 0 & 59 \\
\hline Culturas temporárias & 1529 & 205 & 0 & 7 & 18872 & 2432 & 13666 & 8047 & 4644 & 3 & 49405 \\
\hline Culturas permanentes & 75 & 0 & 0 & 0 & 27 & 1591 & 2195 & 710 & 580 & 0 & 5178 \\
\hline Áreas agrícolas heterogéneas & 2531 & 40 & 4 & 0 & 7876 & 1569 & 9717 & 11427 & 2934 & 0 & 36098 \\
\hline Florestas & 1175 & 364 & 2 & 3 & 1917 & 1033 & 2639 & 50314 & 37619 & 105 & 95171 \\
\hline $\begin{array}{l}\text { Florestas abertas e vegetação } \\
\text { arbustiva e herbácea }\end{array}$ & 444 & 71 & 27 & 0 & 977 & 1035 & 1166 & 10838 & 80640 & 915 & 96113 \\
\hline $\begin{array}{l}\text { Áreas descobertas e com pouca } \\
\text { vegetação }\end{array}$ & 25 & 29 & 0 & 0 & 78 & 94 & 199 & 8056 & 27849 & 1350 & 37680 \\
\hline Total cos07 & 9821 & 826 & 33 & 30 & 30781 & 7908 & 30723 & 90029 & 154629 & 2382 & 684840 \\
\hline
\end{tabular}

